\title{
Stability of 2D natural convection flows in air-filled differentially heated cavities: 2D/3D Disturbances
}

\author{
Shihe Xin ${ }^{1} \ddagger$ and Patrick Le Quéré ${ }^{2} \S$ \\ ${ }^{1}$ Insa-Lyon, Cethil, UMR5008, 21 av. Albert Einstein 69621 Villeurbanne Cedex, \\ France \\ ${ }^{2}$ LIMSI-CNRS, BP 133, 91403 Orsay Cedex, France
}

\begin{abstract}
Further to previous 2D studies of flows in differentially heated cavities filled with air, we are interested in stability of $2 \mathrm{D}$ natural convection flow in these cavities with respect to 3D periodic perturbations. The basis of the numerical methods is a time-stepping code using Chebyshev spectral collocation method and direct Uzawa method for velocity-pressure coupling. Newton's iteration, Arnoldi's method and continuation method have been used in order to respectively compute 2D steady-state base solution, estimate the leading eigenmodes of the Jacobian and perform linear stability analysis. Differentially heated air-filled cavities of aspect ratios from 1 to 7 were investigated. Neutral curves (Rayleigh number versus wave number) have been obtained. It turned out that, only for aspect ratio 7, 3D stationary instability occurs at slightly higher Rayleigh numbers than the onset of $2 \mathrm{D}$ time-dependent flow and that for other aspect ratios 3D instability always takes place before 2D time-dependent flows. 3D unstable modes are stationary and anti-centro-symmetric. 3D nonlinear simulations revealed that the corresponding pitchfork bifurcations are supercritical and that 3D instability leads only to weak flow in the third direction. Further 3D computations are also performed at higher Rayleigh number in order to understand the effects of the weak 3D fluid motion on the onset of time-dependent flow. 3D flow structures are responsible for the onset of time-dependent flow for aspect ratios 1, 2 and 3 while for larger aspect ratios they do not alter the transition scenario which was observed in $2 \mathrm{D}$ cases and that vertical boundary layers become unstable to traveling waves.
\end{abstract}

Keywords: Natural convection, Stability analysis, Air-filled cavity, Spectral methods

$\ddagger$ Corresponding author: shihe.xin@insa-lyon.fr

$\S$ plq@limsi.fr 


\section{Introduction}

The onset of time-dependent flows and transition to chaos of natural convection in 2D differentially heated cavities has been the subject of intensive research in the past decades (see (Paolucci \& Chenoweth 1989, Le Quéré 1987, Le Quéré \& Behnia 1998, Paolucci \& Chenoweth 1989, Xin \& Le Quéré 1995, Mercader et al. 2005) among others). Although there are more and more 3D investigations of natural convection in cavities, it is still interesting and necessary to understand the validity of $2 \mathrm{D}$ flow assumption. To this purpose we performed linear stability analysis of steady 2D natural convection flows with respect to 3D disturbances.

In general natural convection flows were investigated by time-stepping codes (using a time scheme to solve the unsteady Navier-Stokes equations). But time-stepping codes are not able to obtain unstable steady flows which are base solutions of linear stability analysis. Recently iterative techniques (Newton's iteration, approximate exponentiation of the Jacobian and Arnoldi's method) making use of time-stepping codes have been proposed (Mamun \& Tuckerman 1995, Tuckerman \& Barkley 2000) for steady-state solving and linear stability analysis. We combined them with continuation method and secant method to perform linear stability analysis of a two-dimensional base solution with respect to both two- and tree-dimensional perturbations (Xin \& Le Quéré 2001): the codes are based on spectral Chebyshev collocation in space and a differentially heated square cavity with conducting horizontal walls was revisited. The main conclusion is that 2D flow assumption is valid for high Prandtl number as for small Prandtl number the $2 \mathrm{D}$ base solution becomes unstable to $3 \mathrm{D}$ disturbances.

In a recent study (Xin \& Le Quéré 2006) we revisited the onset of time-dependent flows in air-filled cavities with adiabatic horizontal walls: accurate critical values of Rayleigh number and angular frequency have been determined and furthermore multiple steady solutions have been discovered for aspect ratio (height/width) about 3 by combining steady-state solving with quadratic extrapolation.

In the present work we are interested in differentially heated air cavities with adiabatic horizontal walls and, in particular, the stability of 2D steady-state solutions with respect to $3 \mathrm{D}$ disturbances. The height/width aspect ratios considered range from 1 to 7 . Linear stability analysis is first performed with respect to 3D disturbances (3D stationary disturbances are more unstable than the $2 \mathrm{D}$ ones except for 7 ), 3D nonlinear simulations are then realized for slightly supercritical Rayleigh numbers in order to understand the behavior of the bifurcated 3D steady solutions and further 3D computations are finally conducted for higher Rayleigh numbers so as to locate the transition to time-dependent flow and know the influence of the weak 3D flows on the transition mechanisms.

In the following we first specify the configuration of the physical problem investigated, then give a brief description of numerical methods used and discuss the numerical results obtained before giving finally the concluding remarks. 


\section{Physical problem and governing equations}

We are interested in air flows in differentially heated cavities with adiabatic horizontal walls and suppose that they are governed by the Navier-Stokes equations under Boussinesq assumption. Before investigating directly 3D configurations, the problem of interest is divided into two distinguished but simpler configurations: a well-known 2D one is governed by the 2D Navier-Stokes equations and a 3D one is governed by the linearized 3D Navier-Stokes equations of periodic disturbances in $y$ direction.

The 2D configuration is an air-filled cavity of height $H$ and width $W$ in the $x-z$ plane. Its vertical walls are submitted to a constant temperature difference $\Delta T=T_{h}-T_{c}$ and its horizontal walls are adiabatic. It depends on Rayleigh number $\left(R a=\left(g \beta \Delta T H^{3}\right) /(\nu \alpha)\right.$, Prandtl number $(\operatorname{Pr}=\nu / \alpha, 0.71$ for air flow $)$ and aspect ratio $(A=H / W)$ where $g, \beta, \nu$ and $\alpha$ represent respectively gravity acceleration, thermal expansion coefficient, kinematic viscosity and thermal diffusivity. Note that in 3D nonlinear cases $A=H / W$ will be denoted by $A_{x}$. Using the following reference quantities: $H$ for length, $\alpha R a^{1 / 2} / H$ for velocity and $H^{2} R a^{-1 / 2} / \alpha$ for time and defining a reduced temperature $\Theta=\left(T-T_{0}\right) / \Delta T$ with $T_{0}=\left(T_{h}+T_{c}\right) / 2$, the unsteady $2 \mathrm{D}$ Navier-Stokes equations read in dimensionless form :

$$
\begin{aligned}
0 & =\frac{\partial U}{\partial x}+\frac{\partial W}{\partial z} \\
\frac{\partial U}{\partial t}+U \frac{\partial U}{\partial x}+W \frac{\partial U}{\partial z} & =-\frac{\partial P}{\partial x}+\frac{P r}{R a^{0.5}}\left(\frac{\partial^{2}}{\partial x^{2}}+\frac{\partial^{2}}{\partial z^{2}}\right) U \\
\frac{\partial W}{\partial t}+U \frac{\partial W}{\partial x}+W \frac{\partial W}{\partial z} & =-\frac{\partial P}{\partial z}+\frac{P r}{R a^{0.5}}\left(\frac{\partial^{2}}{\partial x^{2}}+\frac{\partial^{2}}{\partial z^{2}}\right) W+\operatorname{Pr} \Theta \\
\frac{\partial \Theta}{\partial t}+U \frac{\partial \Theta}{\partial x}+W \frac{\partial \Theta}{\partial z} & =\frac{1}{R a^{0.5}}\left(\frac{\partial^{2}}{\partial x^{2}}+\frac{\partial^{2}}{\partial z^{2}}\right) \Theta
\end{aligned}
$$

The boundary conditions are no-slip for velocity and $\Theta(0, z)=0.5, \Theta(1 / A, z)=-0.5$ and adiabatic on horizontal walls for temperature, i.e. $\frac{\partial \Theta}{\partial z}(x, 0)=\frac{\partial \Theta}{\partial z}(x, 1)=$ 0. $2 \mathrm{D}$ solutions verify the following centro-symmetry (CS): $(\Theta, U, W)(x, z)=$ $-(\Theta, U, W)(1 / A-x, 1-z)$.

Periodic 3D disturbances characterized by $k$, a wave number in the third direction ( $y$ direction), are then governed by the 3D Navier-Stakes equations linearized about $(\Theta, U, W)$. 3D periodic perturbations are written as follows: $\theta(x, z) \cos (k y)$, $u(x, z) \cos (k y), \quad v(x, z) \sin (k y)$ and $w(x, z) \cos (k y)$. The eigenmodes are centrosymmetric (CS) when $(\theta, u, v, w)(x, z)=(-\theta,-u, v,-w)(1 / A-x, 1-z)$ and anticentro-symmetric (ACS) when $(\theta, u, v, w)(x, z)=(\theta, u,-v, w)(1 / A-x, 1-z)$. If $3 \mathrm{D}$ disturbances are amplified in time, $2 \mathrm{D}$ base solution is unstable with respect to periodic 3D disturbances; on the other hand if they are damped in time, it is stable. In this way one can perform linear stability analysis of a $2 \mathrm{D}$ base solution with respect to periodic 3D disturbances.

Finally, solving the 3D unsteady Navier-Stokes equations is necessary to understand nonlinear behavior of the bifurcations related to 3D periodic perturbations and perform numerical study at higher Rayleigh numbers. The linearized and fully 3D Navier-Stakes 
equations are omitted here and details can be found in (Xin \& Le Quéré 2001, Xin \& Le Quéré 2002).

\section{Numerical methods}

All of our codes use spectral Chebyshev collocation methods and Fourier series for spatial discretization (Canuto et al. 1988, Bernardi \& Maday 1992, Peyret 2000, Boyd 2000). Direct Uzawa method (Canuto et al. 1988, Bernardi \& Maday 1992) is employed to ensure fluid incompressibility (velocity-pressure coupling) except for the 3D nonlinear cases in which projection method (Guermond \& Quartapelle 1998) is used.

\subsection{D time-stepping code}

Equations (1) are traditionally solved by time-stepping codes making use of time schemes. These 2D codes have been widely used to study the onset of time-dependent flows in cavities and are also used in the present work in order to initialize steady-state solving through Newton's iteration. The time-stepping code combines a second-order Backward Differencing Formula (BDF2) for linear part with a second-order AdamsBashforth extrapolation for the convective terms. The resulted 2D Helmholtz equations for the unknowns are solved by a direct method based on full diagonalization of the second-order partial derivatives (Haidvogel \& Zang 1979).

\subsection{Steady-state solving through Newton's iteration}

Time-stepping codes, though widely used, suffer from several drawbacks for performing stability analysis. One should resort to Newton's iteration to obtain unstable steadystate solutions and fortunately iterative techniques for solving Newton's iteration, avoiding explicit building of the Jacobian, are now available (Mamun \& Tuckerman 1995, Tuckerman \& Barkley 2000). These techniques have been coded and applied successfully to natural convection flows in (Xin et al. 1997, Xin \& Le Quéré 2001, Xin \& Le Quéré 2002).

\subsection{Linear stability analysis}

Once a steady-state 2D solution is obtained, its linear stability can be investigated by integrating, with very small time step, the linearized 3D Navier-Stokes equations and using Arnoldi's method as solution at one time step is the action of the approximate exponential transformation of the Jacobian on the solution at a previous time step. A large number of iterations is generally needed to separate the eigenmodes due to very small time-step and the corresponding computation may be still expensive.

One idea for improving accuracy and efficiency of the computations is to use the results of Arnoldi's method as initial estimates of the eigenmodes and work, by using a continuation technique, on the eigensystem (Keller 1977). This approach was already 
followed in (Winters 1987). We propose to apply it to the eigensystem governing 3D periodic disturbances and details can be found in (Xin \& Le Quéré 2001, Xin \& Le Quéré 2002).

\subsection{D time-stepping code}

Once critical values corresponding to 3D perturbations are calculated accurately, 3D time-stepping codes are needed to study the nature of instabilities induced by the 3D eigenmodes and the nonlinear behavior of the critical mode. The 3D time-stepping code uses also a second-order Backward Differencing Formula (BDF2) and the resulted 3D Helmholtz equations are also solved by a direct method (Haidvogel \& Zang 1979).

\subsection{Convergence criteria and accuracy}

The convergence criterion used for Newton's iteration is that the $\|\cdot\|_{\infty}$ of the correction becomes smaller than $10^{-7}$. Its quadratic convergence in the neighborhood of the searched root means that $\|\cdot\|_{\infty}$ of the next solution increment will be at round-off level. Discrete steady-state dimensionless solutions whose ranges lie in $[-0.5,0.5]$ for reduced temperature and $(-0.25,0.25)$ for dimensionless velocity field are therefore converged to round-off level.

Continuation method applied to the eigensystem is also solved by Newton's iteration and the convergence criterion is also that $\|\cdot\|_{\infty}$ of the correction be smaller than $10^{-7}$ : the corresponding change in $R a$ is smaller than 10. But due to the computation cost, a coarse resolution in $k$ was used and critical Rayleigh number is only given with 4 significant figures.

For fully 3D numerical simulations, accuracy of numerical solutions can be measured by spectral coefficients of the highest polynomial degrees of temperature field, for example. Their $\|\cdot\|_{\infty}$ in each direction have been checked : in the periodic $y$ direction $\|\cdot\|_{\infty} \leq 10^{-10}$ and in the other two Chebyshev directions $\|\cdot\|_{\infty} \leq 10^{-5}$.

\section{Results and discussions}

Generally speaking, linear stability analysis of a 2D base solution can be performed for either $2 \mathrm{D}$ or $3 \mathrm{D}$ periodic disturbances. The onset of $2 \mathrm{D}$ time-dependent flows is characterized by $2 \mathrm{D}$ oscillatory perturbations.

In order to illustrate the difference between 2D and 3D periodic disturbances, we recall first critical values corresponding to the onset of $2 \mathrm{D}$ time-dependent flows and present then numerical results obtained for 3D periodic perturbations.

\subsection{D perturbations: onset of time-dependent flow}

The onset of time-dependent flows in differentially heated air-filled cavities has been intensively studied by time stepping codes. But critical values determined in this way 
Table 1. Critical values of $2 \mathrm{D}$ oscillatory and $3 \mathrm{D}$ stationary disturbances versus aspect ratio, $A$. $R a_{c}^{2 D}$-critical Rayleigh number of $2 \mathrm{D}$ modes, $\omega_{c}$-angular frequency of the $2 \mathrm{D}$ critical mode, $R a_{c}^{3 D}$-critical Rayleigh number of $3 \mathrm{D}$ modes, $k_{c}$-critical wave number in $y$ direction, ACS-Anti-Centro-Symmetry, CS-Centro-Symmetry.

\begin{tabular}{llllllll}
\hline & \multicolumn{3}{c}{ 2D oscillatory disturbances } & \multicolumn{4}{c}{ 3D stationary disturbances } \\
\hline$A$ & $R a_{c}^{2 D}$ & $\omega_{c}$ & Symmetry & $N I \times N K$ & $R a_{c}^{3 D}$ & $k_{c}$ & Symmetry \\
\hline 1 & $1.825 \times 10^{8}$ & 0.2930 & ACS & $100 \times 100$ & $1.5504 \times 10^{7}$ & 19.2 & ACS \\
2 & $1.587 \times 10^{8}$ & 0.2823 & ACS & $80 \times 100$ & $1.637 \times 10^{7}$ & 19.1 & ACS \\
3 & $1.113 \times 10^{8}$ & 0.3327 & ACS & $40 \times 80$ & $1.140 \times 10^{7}$ & 18.5 & ACS \\
4 & $1.032 \times 10^{8}$ & 2.5399 & CS & $40 \times 80$ & $2.141 \times 10^{7}$ & 22.7 & ACS \\
5 & $0.970 \times 10^{8}$ & 2.7940 & CS & $40 \times 80$ & $4.180 \times 10^{7}$ & 26.75 & ACS \\
6 & $1.109 \times 10^{8}$ & 3.4752 & CS & $40 \times 90$ & $7.628 \times 10^{7}$ & 30.90 & ACS \\
7 & $1.261 \times 10^{8}$ & 3.9353 & ACS & $40 \times 90$ & $1.274 \times 10^{8}$ & 34.70 & ACS \\
8 & $1.568 \times 10^{8}$ & 4.0732 & ACS & & & & \\
\hline
\end{tabular}

are not accurate. We revisited recently these cavities by performing linear stability analysis for aspect ratios ranging from 1 to 8 and provided accurate critical values (Xin \& Le Quéré 2002, Xin \& Le Quéré 2006). Table 1 summarizes the results obtained.

Critical Rayleigh numbers of 2D disturbances listed in Table 1 are all of order $10^{8}$ and critical angular frequencies divide the cavities into two groups: One group of low aspect ratios with low angular frequency and another of high aspect ratio with higher angular frequency. With low $\omega_{c}$ the critical modes fill almost completely the cavities and are due to flow structure at the exiting corners of vertical boundary layers, which has been called internal hydraulic jump. The critical modes of higher angular frequency are of boundary layer type and are located mainly along the cavity walls. In terms of symmetry, 2D steady-state base solutions are centro-symmetric (CS), some critical modes keep this symmetry and some break it.

Note that for aspect ratio about 3 there were observed multiple steady-state solutions. For certain aspect ratios between 3 and 4 there exists two critical Rayleigh numbers, one per solution branch. More details can be found in (Xin \& Le Quéré 2006).

\subsection{D perturbations: linear stability}

One of the difficulties encountered in studying 3D perturbations is that there is one more unknown, the wave number $k$. As there is no a priori information about the critical Rayleigh number and critical wave number, the only way to do it is the test-and-error method: after obtaining a steady-state solution at a fixed Rayleigh number, the 3D linearized Navier-Stokes equations are integrated in time for several wave numbers in order to observe whether perturbations are amplified. Once a wave number for which 3D perturbations are amplified in time is found, we calculate the critical Rayleigh number for the wave number considered and construct neutral curves versus wave number. In order to make sure that the minima of these neutral curves correspond really to the 
critical Rayleigh numbers, one must check that there is no more unstable modes below these minima.

In a previous study (Xin \& Le Quéré 2002) air-filled cavity of aspect ratio 8 has been investigated in detail. It was shown that air flow in this cavity becomes first unstable to 2D oscillatory perturbations and that 3D periodic perturbations are less dangerous than $2 \mathrm{D}$ perturbations. Therefore it is meaningful to perform 2D analysis for air-filled cavity of aspect ratio 8 at least up to the onset of time-dependent flows. For this aspect ratio it is the $2 \mathrm{D}$ time-dependent solutions that become unstable to $3 \mathrm{D}$ periodic disturbances in $y$ direction (Xin \& Le Quéré 2002).
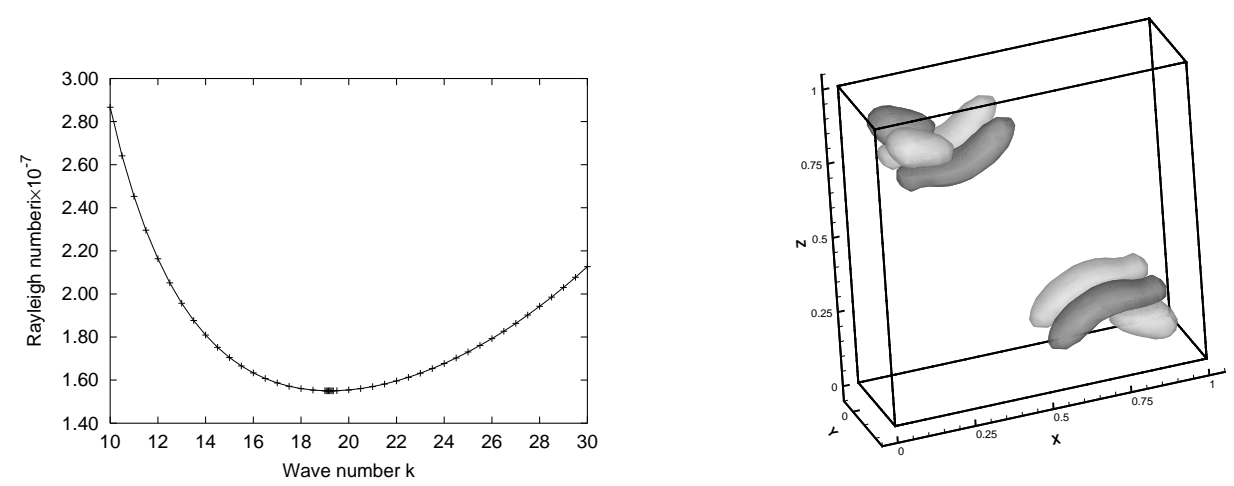

Figure 1. Neutral curve of the 3D unstable mode (left) and iso-surfaces of eigentemperature (right) obtained for 3D periodic perturbations in $y$ direction in a square cavity $(A=1)$. Critical values are $R a_{c}^{3 D}=1.55 \times 10^{7}$ and $k_{c}=19.2$, the unstable mode is stationary and it corresponds to a pitchfork bifurcation.

In this work, aspect ratios from 1 to 7 were investigated in detail. It turned out that in a square cavity $3 \mathrm{D}$ periodic stationary perturbations are more dangerous than 2D oscillatory perturbations. We display in Figure 1 the neutral curve and the corresponding eigen-temperature obtained with a spatial resolution of $70 \times 70$. The critical Rayleigh number is equal to $1.550 \times 10^{7}$ and the critical wave number 19.2. Spatial distribution of eigen-temperature indicates that the critical mode is anti-centrosymmetric (ACS, i.e. breaking the symmetry of base solution) and linked to the flow structure at the exiting corners of the vertical boundary layers as was the case for $2 \mathrm{D}$ oscillatory perturbations. But the 3D periodic critical mode is stationary (zero angular frequency). Note also that the critical Rayleigh number of 3D periodic perturbations is one order of magnitude smaller than the one of $2 \mathrm{D}$ oscillatory perturbations. In order to know grid dependence of the results, a higher spatial resolution of $100 \times 100$ was used: at $k=19.2$ the critical Rayleigh number is equal to $1.5504 \times 10^{7}$, which confirms the accuracy of the results obtained with a grid $70 \times 70$.

A spatial resolution of $80 \times 100$ was used to investigate the cavity of aspect ratio $A=2$. Critical Rayleigh number and wave number are equal to respectively $1.637 \times 10^{7}$ and 19.1. The critical mode is also stationary and anti-centro-symmetric, its structure is similar to that found in a square cavity (Figure 2). 

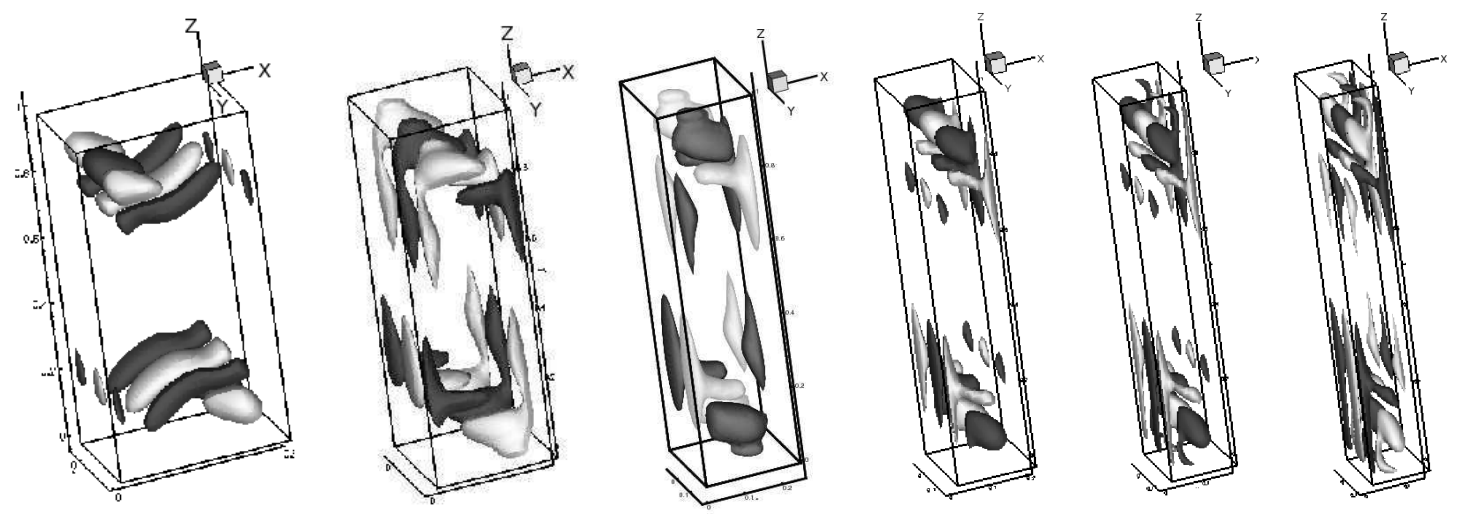

Figure 2. 3D critical modes (eigen-temperature) obtained for $A=2,3,4,5,6$, and 7 (from left to right). The unstable modes are concentrated in the top and bottom parts and this indicates that the corresponding instabilities are related to horizontal flow in these cavities.
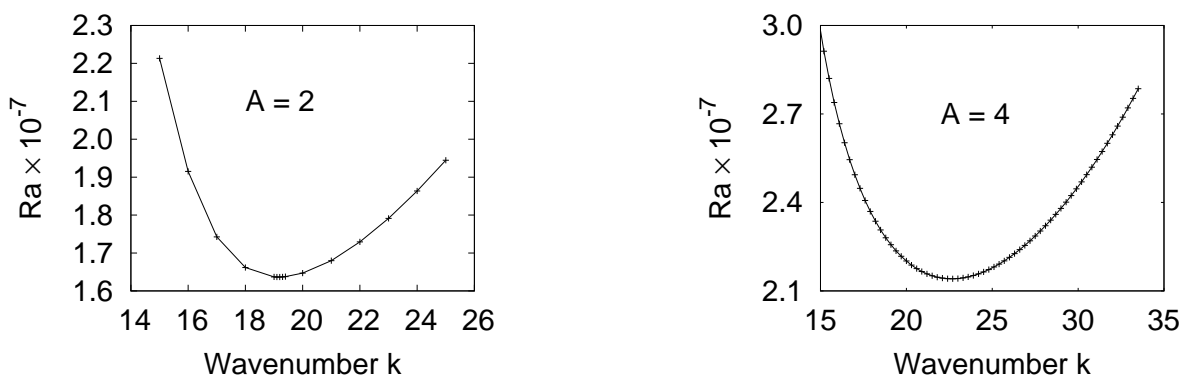

Figure 3. Examples of neutral curves of the 3D unstable modes obtained for 3D periodic perturbations in $y$ direction.

The cavities of $A=3$ and 4 were studied using a grid of $40 \times 80$. For $A=3$, the critical wave number, $k_{c}$, is equal to 18.5 and the critical Rayleigh number, $R a_{c}^{3 D}$, is equal to $1.140 \times 10^{7}$. For a cavity of $A=4, k_{c}=22.7$ and $R a_{c}^{3 D}=2.141 \times 10^{7}$. The corresponding critical modes are also stationary and have the anti-centro-symmetry which breaks the centro-symmetry of the $2 \mathrm{D}$ base solution.

Grids used to investigate $A=5,6$ and 7 were respectively $40 \times 80,40 \times 90$ and $40 \times 90$. The critical wave numbers are equal to respectively $26.75,30.90$ and 34.70 . The corresponding critical Rayleigh numbers are equal to $4.180 \times 10^{7}, 7.628 \times 10^{7}$ and $1.274 \times 10^{8}$. It is to note that these stationary critical modes are really similar in terms of spatial structure and that they are all anti-centro-symmetric.

The spatial resolutions used above are inspired by the previous $2 \mathrm{D}$ works (Xin \& Le Quéré 2001, Xin \& Le Quéré 2002, Xin \& Le Quéré 2006). In fact, for $1 \leq A \leq 8$, heat transfer and boundary layer thickness depend only on the Rayleigh number based on cavity height. When working on a particular $R a$ based on cavity height, one can use less collocation points in $x$ direction for larger $A$ : this is why with increasing $A$ less collocation points are used. As 70 or 80 collocation points in $z$ direction are enough for $A=1$ and 2 at $R a \approx 10^{7}, 80$ collocation points in $z$ direction are kept for $A=3,4$ and 
5. Because $R a_{c}^{3 D}$ increases rapidly with higher $A$ and boundary layer becomes thinner, higher spatial resolution is used in $z$ direction while 40 points are kept in $x$ direction.

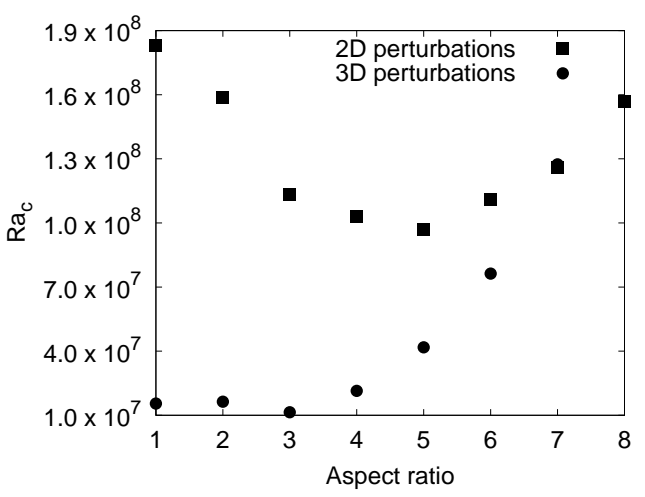

Figure 4. Comparison of the critical Rayleigh numbers established for 2D and 3D perturbations and aspect ratios between 1 and 8 . 3D periodic perturbations are more unstable for $A \leq 6$ and 3D simulations are thus necessary.

Table 1 summarizes also spatial resolution used and important features of the numerical results obtained for 3D disturbances. More neutral curves are given as examples in Figure 3 and the unstable modes from $A=2$ to $A=7$ are displayed in Figure 2 in terms of eigen-temperature. For the aspect ratios investigated all the 3D periodic modes are found to be stationary and anti-centro-symmetric and they correspond to therefore pitchfork bifurcations. The critical modes of $A=1$ and 2 have similar spatial structure which is due to the so-called internal hydraulic jump at the exiting corners of the vertical boundary layers and the critical values remain almost constant. For larger aspect ratios, the critical 3D modes are also related to flow structures near the horizontal walls, both the critical Rayleigh number and the critical wave number increase with aspect ratio. All the critical Rayleigh numbers of 3D modes are smaller than those of 2D oscillatory perturbations (Table 1) except for the aspect ratio 7 (Figure 4). This means that, for $A \leq 6,2 \mathrm{D}$ steady natural convection flows in differentially heated air-filled cavities will become steady and three-dimensional before becoming time-dependent if there is enough space in $y$ direction. These results indicate that future 2D studies should be conducted carefully in order to remain meaningful.

As all the 3D unstable modes are concentrated on the top and bottom parts in the cavities, they are related to the flow structure there. The common flow feature in the cavity top parts is that fluid goes up, turns around the corner, follows the horizontal wall and joins the downward boundary layer. This takes place smoothly for $A=3-7$ but with an internal hydraulic jump for $A=1$ and 2: after turning around the corner, fluid rebounds first downwards and moves then upwards while flowing towards the cold wall. In these flows fluid particles follow curving trajectories and a possible instability mechanism is centrifugal-flow instability because fluid particles are submitted to centrifugal force. Generally speaking, centrifugal force results in curvature deformation in the transverse direction and centrifugal-flow instability corresponds to 
Table 2. Slightly supercritical Rayleigh numbers investigated and numerical parameters used for $3 \mathrm{D}$ simulations. Aspect ratio- $A_{x}$, wave length- $A_{y}=2 \pi / k$, spatial resolution- $N I \times N J \times N K$.

\begin{tabular}{ccccc}
\hline $\begin{array}{c}\text { Aspect } \\
\text { ratio }\left(A_{x}\right)\end{array}$ & $\begin{array}{c}\text { Rayleigh } \\
\text { number }\end{array}$ & $\begin{array}{c}\text { Wave length } \\
\text { (wave number) }\end{array}$ & $\begin{array}{c}\text { Spatial } \\
\text { Resolution }\end{array}$ & $\begin{array}{c}\text { Time } \\
\text { step }\end{array}$ \\
\hline 1 & $1.8 \times 10^{7}$ & $0.33(19.04)$ & $80 \times 30 \times 80$ & $1 \times 10^{-2}$ \\
2 & $1.8 \times 10^{7}$ & $0.33(19.04)$ & $60 \times 30 \times 80$ & $1 \times 10^{-2}$ \\
3 & $1.4 \times 10^{7}$ & $0.34(18.48)$ & $40 \times 30 \times 80$ & $1.5 \times 10^{-2}$ \\
4 & $2.5 \times 10^{7}$ & $0.28(22.44)$ & $40 \times 30 \times 80$ & $1.4 \times 10^{-2}$ \\
5 & $5.0 \times 10^{7}$ & $0.24(26.18)$ & $40 \times 30 \times 80$ & $1 \times 10^{-2}$ \\
6 & $8.5 \times 10^{7}$ & $0.20(31.42)$ & $50 \times 30 \times 90$ & $1 \times 10^{-2}$ \\
\hline
\end{tabular}

secondary flow of the main flow. Centrifugal-flow instability does occur in cavity flows: it is well-known in lid-driven cavities (see (Ramanan \& Homsy 1994, Albensoeder et al. 2001) among others) and it is also observed in a differentially heated square cavity filled with low-Pr fluids (Xin \& Le Quéré 2001). Figure 2 indicates clearly that 3D unstable modes are secondary flow of the main flow stream and that they are centered on the curving main flow. For $A=1$ and 2 the main flow curvature is particular due to the internal hydraulic jump and the unstable modes follow exactly this curving flow structure. For $A \geq 3$ curvature of the main flow is modified and the unstable modes display then a different structure. This change in structure can also explain the brutal change observed in $R a_{c}^{3 D}$ between $A=2$ and $A=3$ (Figure 4).

\subsection{D perturbations: nonlinear behavior}

In order to understand nonlinear behavior of the 3D unstable modes, 3D numerical simulations using 3D time-stepping code were performed and randomly perturbed 2D base solutions were used as initial conditions. Table 2 summarizes Rayleigh numbers studied and the corresponding numerical parameters. Note that two aspect ratios, $A_{x}=H / W$ ( $A$ in $2 \mathrm{D}$ cases) and $A_{y}=2 \pi / k$ (wave length), are used for these 3D simulations and that spatial resolution corresponds to $N I \times N J \times N K$ in respectively $x, y$ and $z$ directions.

Steady-state solutions after bifurcations are displayed in Figures 5 and 6 in terms of iso-surfaces of temperature and $u$ velocity. As predicted by linear stability analyses performed, 3D structures are located in the top and bottom regions. As base solutions are only 2D ( $V$ is thus equal to zero), in full 3D cases $v$ velocity is simply perturbations and it can represent the amplitude of the unstable mode. Its time evolution gives an insight into signs of the coefficients in the normal-form model of a pitchfork bifurcation. Time evolutions of $v$ velocity indicate that all the pitchfork bifurcations are supercritical: an example with $A_{x}=4$ is given in Figure 7 . Note nevertheless that $3 \mathrm{D}$ flows are very weak in $y$-direction: $v$ velocity is one order of magnitude smaller than $u$ and $w$ velocities that lie in respectively $[-0.1,0.1]$ and $[-0.22,0.22]$. These pitchfork bifurcations have 

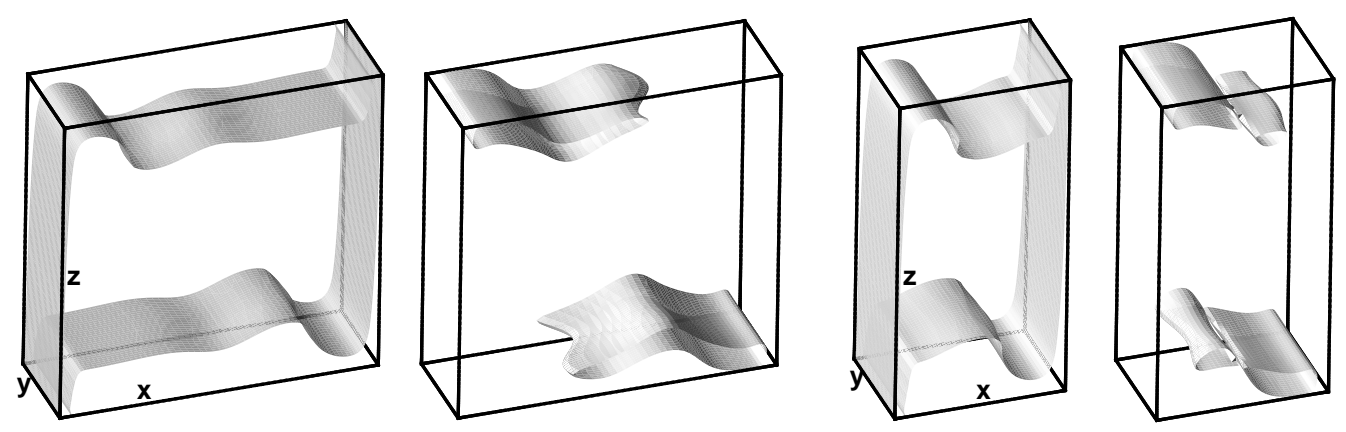

Figure 5. Iso-surfaces of temperature and $u$ velocity obtained by 3D time-stepping simulations for $A_{x}=1$ and $A_{y}=0.33$ at $R a=1.8 \times 10^{7}$ (left) and $A_{x}=2$ and $A_{y}=0.33$ at $R a=1.8 \times 10^{7}$ (right)

only weak influence on the global flow structure and heat transfer.
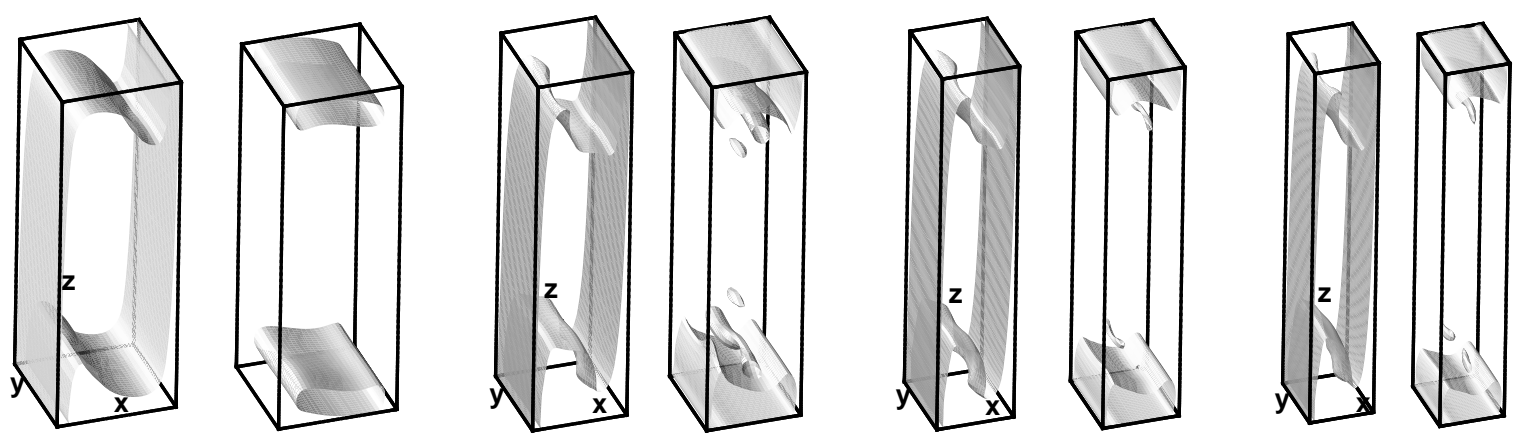

Figure 6. 3D nonlinear results in terms of iso-surfaces of temperature and $u$ velocity for $A_{x}=3\left(R a=1.4 \times 10^{7}\right.$ and $\left.A_{y}=0.34\right), A_{x}=4\left(R a=2.5 \times 10^{7}\right.$ and $\left.A_{y}=0.28\right)$ $A_{x}=5\left(R a=5 \times 10^{7}\right.$ and $\left.A_{y}=0.24\right)$ and $A_{x}=6\left(R a=8.5 \times 10^{7}\right.$ and $\left.A_{y}=0.20\right)$ (from left to right).
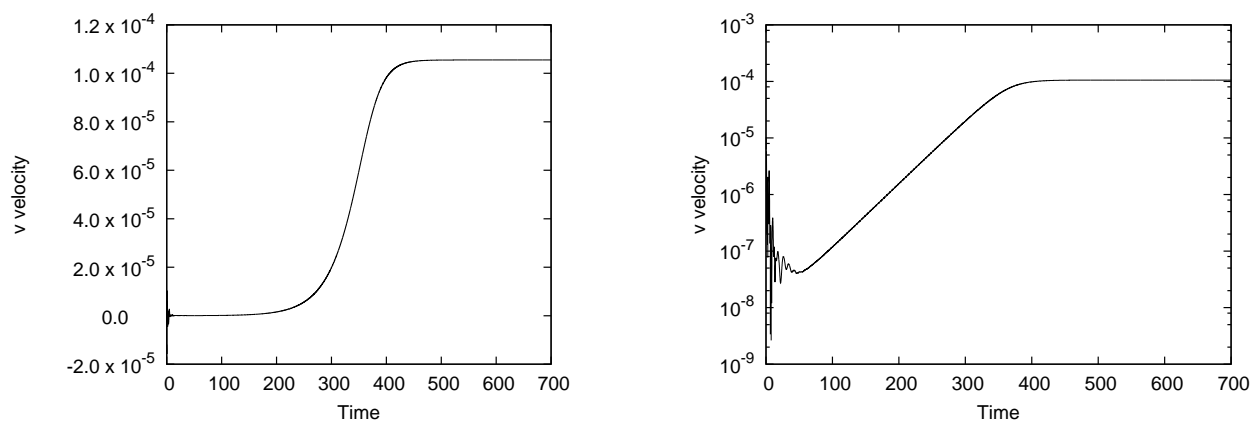

Figure 7. Time evolution of $v$ velocity (left) and the corresponding log-scale display (right) in a cavity of $A_{x}=4 . v$ velocity represents perturbations and the pitchfork bifurcation is supercritical. The same stands also for other $A_{x}$.

\subsection{D time-dependent flow}

As the 3D unstable modes result in weak 3D flows at slightly supercritical Rayleigh number, an interesting question is to know whether these weak flows persist at higher 
Table 3. Summary of the transition to time-dependent 3D flow. Aspect ratio- $A_{x}$, aspect ratio- $A_{y}$, spatial resolution- $N I \times N J \times N K, R a_{S}$-the last Rayleigh number at which steady-state solution is obtained, $R a_{T}$-the first Rayleigh number at which timedependent (periodic) solution is observed, П-dimensionless period of time-dependent solution at $R a_{T}$ and $\omega$-the corresponding angular frequency. The transition point is located in $\left[R a_{S}, R a_{T}\right]$.

\begin{tabular}{|c|c|c|c|c|c|c|c|}
\hline$A_{x}$ & $A_{y}$ & $\begin{array}{c}\text { Spatial } \\
\text { Resolution }\end{array}$ & $\begin{array}{l}\text { Time } \\
\text { step }\end{array}$ & $R a_{S}$ & $R a_{T}$ & $\Pi$ & $\omega$ \\
\hline 1 & 0.33 & $80 \times 40 \times 80$ & $8 \times 10^{-3}$ & $4.0 \times 10^{7}$ & $4.5 \times 10^{7}$ & 143.68 & .04373 \\
\hline 2 & 0.33 & $60 \times 40 \times 80$ & $8 \times 10^{-3}$ & $4.0 \times 10^{7}$ & $4.5 \times 10^{7}$ & 389.24 & .01614 \\
\hline 3 & 0.34 & $48 \times 50 \times 80$ & $8 \times 10^{-3}$ & $8.5 \times 10^{7}$ & $9.0 \times 10^{7}$ & 97.08 & .06470 \\
\hline 4 & 0.28 & $40 \times 50 \times 80$ & $5 \times 10^{-3}$ & $1.02 \times 10^{8}$ & $1.05 \times 10^{8}$ & 2.30 & 2.73 \\
\hline 5 & 0.24 & $40 \times 30 \times 80$ & $5 \times 10^{-3}$ & $1.02 \times 10^{8}$ & $1.05 \times 10^{8}$ & 2.27 & 2.76 \\
\hline 6 & 0.20 & $40 \times 30 \times 120$ & $5 \times 10^{-3}$ & $1.1 \times 10^{8}$ & $1.2 \times 10^{8}$ & 1.82 & 3.45 \\
\hline 7 & 0.18 & $40 \times 30 \times 120$ & $5 \times 10^{-3}$ & $1.2 \times 10^{8}$ & $1.3 \times 10^{8}$ & 1.60 & 3.93 \\
\hline
\end{tabular}

$R a$ and understand their effects on the onset of full 3D time-dependent flow. Further 3D nonlinear simulations have thus been conducted at higher $R a$ for $A_{x}=1$ to 7 .

Table 3 summarizes computations performed about the onset of time-dependent $3 \mathrm{D}$ flows. Numerical simulations have been conducted using aspect ratio $A_{y}$ based approximately on the critical wave length $2 \pi / k_{c}$. Critical Rayleigh number is between $4 \times 10^{7}$ and $4.5 \times 10^{7}$ for $A_{x}=1$ and 2 and about $10^{8}$ for other aspect ratios. In terms of period of time-dependent flows, waves of long period (in the order of 100 dimensionless time units !) are observed for small aspect ratios $\left(A_{x}=1,2\right.$ and 3) while for larger aspect ratios waves of short period (in the order of 1 ) are observed.
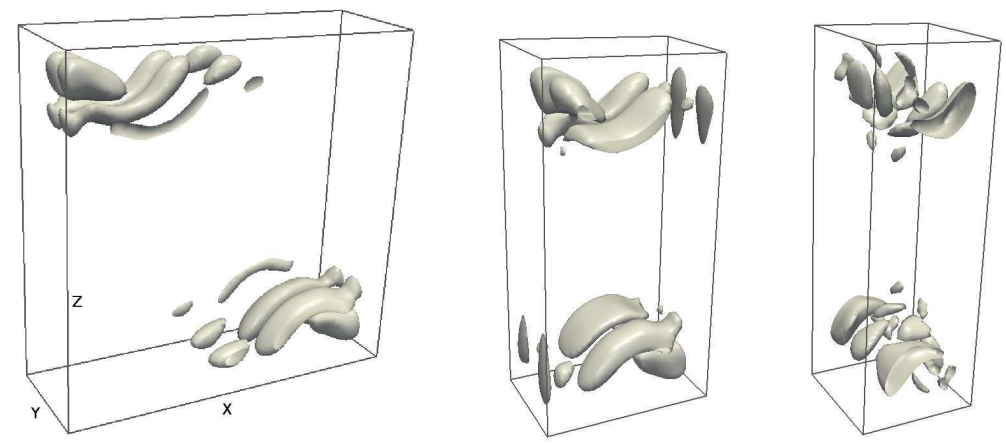

Figure 8. Iso-surfaces of instantaneous temperature fluctuation for $A_{x}=1$ (left, $R a=4.5 \times 10^{7}$ and $A_{y}=0.33$ ), $A_{x}=2$ (middle, $R a=5.0 \times 10^{7}$ and $A_{y}=0.33$ ), and $A_{x}=3$ (right, $R a=9 \times 10^{7}$ and $A_{y}=0.34$ ).

Figure 8 displays instantaneous temperature fluctuations obtained for $A_{x}=1$, 2 and 3 and reveals that temperature fluctuations are located in the flow structure which is called internal hydraulic jump and becomes first unstable to 3D stationary perturbations. Note that for $A_{x}=3$ internal hydraulic jump did not occur at $R a=10^{7}$ when flow becomes $3 \mathrm{D}$. In $2 \mathrm{D}$ case for $A_{x}=3$, internal hydraulic jump takes place 
at higher $R a$ (about $5 \times 10^{7}$ ) through two turning points (Xin \& Le Quéré 2006). This seems to be also the case here for 3D flows (Figure 9 displays multiple solutions obtained at $R a=6 \times 10^{7}$.), but 3D flow structure persists and results in a low frequency Hopf bifurcation. Note also the angular frequencies of these time-dependent flows are much lower than those of the $2 \mathrm{D}$ critical modes (Table 1 ). What happens here is quite similar to the phenomena observed in a differentially heated cubic cavity (Labrosse et al. 1997, de Gassowski et al. 2003) in which very low frequency is responsible for the onset of time-dependent flows. Flow visualization and further computation show that at $A_{x}=1$ and 3 the unstable modes are not traveling waves and that wave periods do not depend on the Rayleigh number, Hopf bifurcations are thus responsible for the onset of time-dependent flow. For $A_{x}=2$ the unstable mode is traveling waves in $y$ direction and its period depends on $R a$ studied (Figure 10). This seems to indicate that the onset of time-dependent flow at $A_{x}=2$ is due to a drift pitchfork bifurcation breaking $O(2)$ symmetry in $y$ direction. As wave speed proportional to $\omega$ depends linearly on $\sqrt{R a-R a_{c}}$ in the case of a supercritical bifurcation, $\omega^{2}$ versus $R a$ is also displayed in Figure 10: the linear relationship confirms a supercritical drift pitchfork bifurcation.
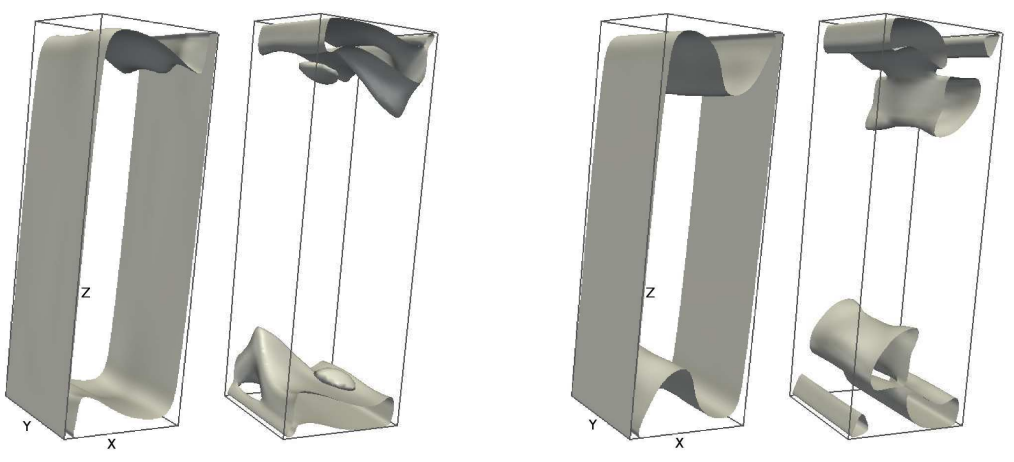

Figure 9. Multiple steady solutions obtained for $A_{x}=3$ at $R a=6 \times 10^{7}$ in terms of iso-surfaces of temperature(first, iso-values of \pm 0.35 ) and $u$ velocity (second, iso-values of \pm 0.02 ): solution without internal hydraulic jump (left) and solution with internal hydraulic jump (right).
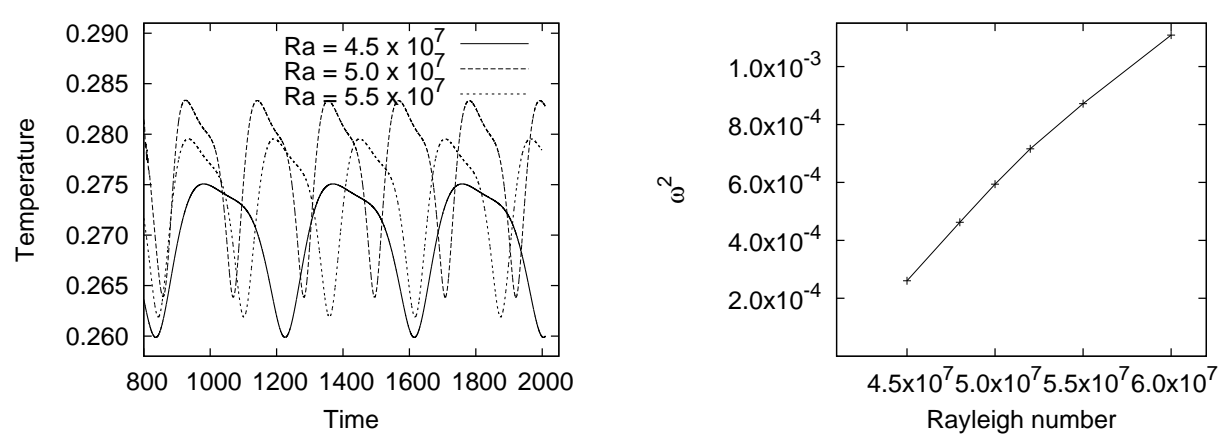

Figure 10. Time evolutions of temperature for several $R a$ (left) and relationship between $\omega^{2}$ and $R a$ (right). The linear relationship between $\omega^{2}$ and $R a$ indicates a supercritical drift pitchfork bifurcation. 

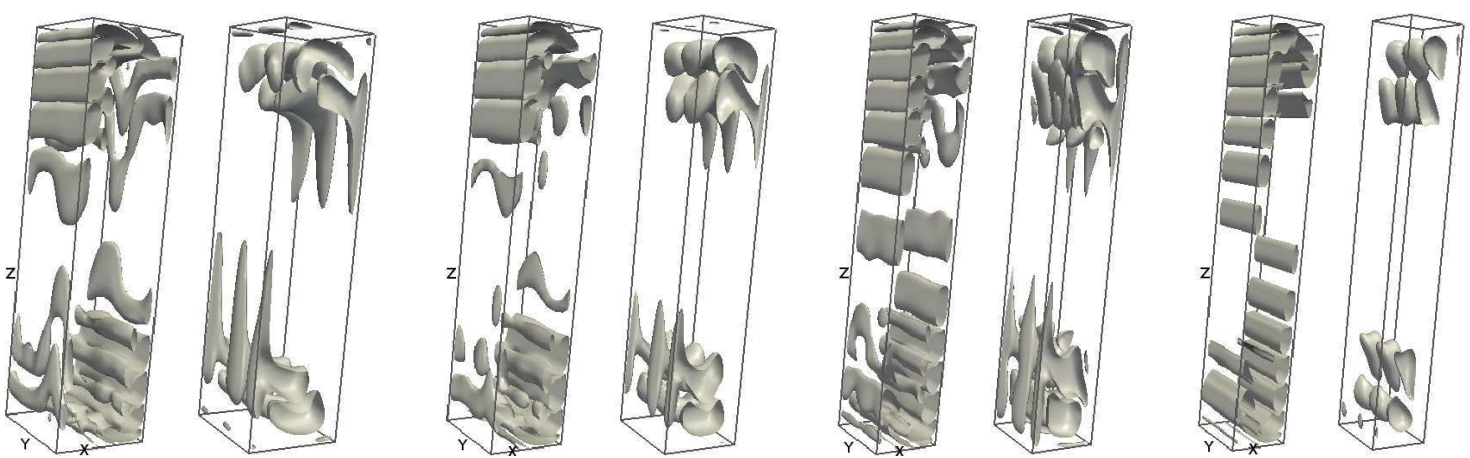

Figure 11. Iso-surfaces of instantaneous temperature fluctuation (first) and $v$ velocity (second) for $A_{x}=4\left(R a=1.05 \times 10^{8}\right.$ and $\left.A_{y}=0.28\right), A_{x}=5\left(R a=1.05 \times 10^{8}\right.$ and $\left.A_{y}=0.24\right), A_{x}=6\left(R a=1.2 \times 10^{8}\right.$ and $\left.A_{y}=0.20\right)$, and $A_{x}=7\left(R a=1.3 \times 10^{8}\right.$ and $A_{y}=0.18$ ) (from left to right). Iso-surfaces of $v$ velocity indicate that $3 \mathrm{D}$ flow persists.
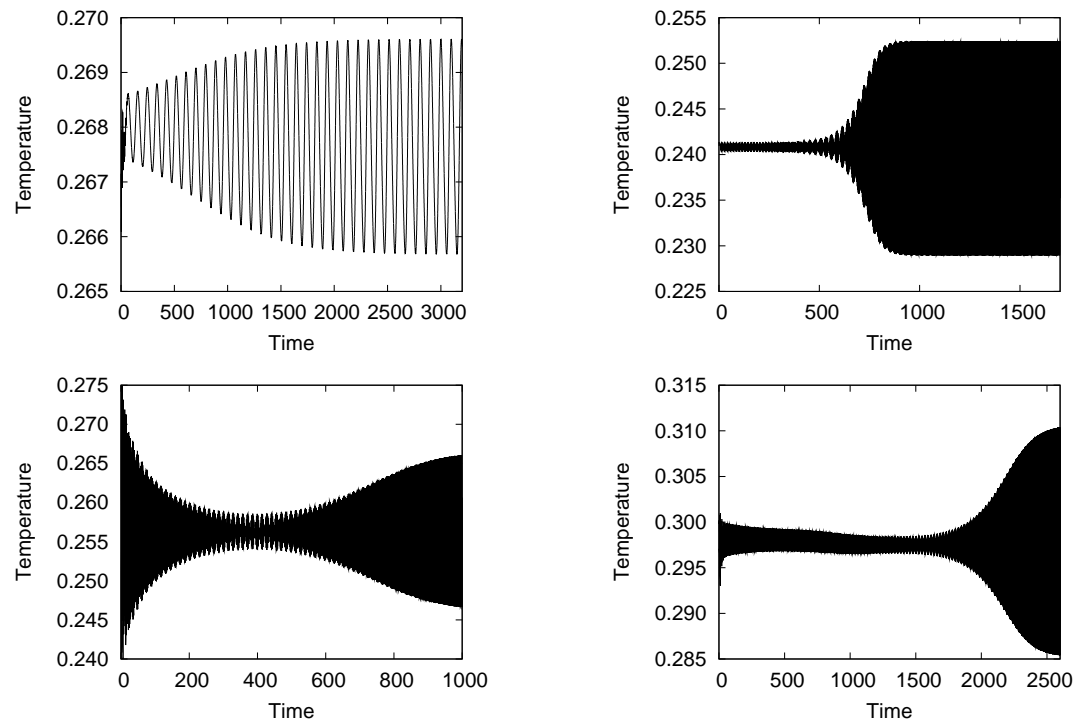

Figure 12. Time evolutions of temperature at a point in the exiting corner of the hot boundary layer. $A_{x}=3$ (top left, $R a=9 \times 10^{7}$ and $A_{y}=0.34$ ), $A_{x}=4$ (top right, $R a=1.05 \times 10^{8}$ and $\left.A_{y}=0.28\right), A_{x}=5$ (bottom left, $R a=1.05 \times 10^{8}$ and $A_{y}=0.24$ ), and $A_{x}=7$ (bottom right, $R a=1.3 \times 10^{8}$ and $A_{y}=0.18$ ).

For larger aspect ratios (from $A_{x}=4$ to 7 ), weak 3D flows persist at higher $R a$ and waves responsible for time-dependent flows are Tollmien-Schlichting waves traveling in the boundary layers along cavity walls (Figure 11), their frequencies (Table 3) are similar to those of the 2D unstable modes (Table 1) and the corresponding critical Rayleigh numbers differ only slightly from those of the 2D perturbations. It means that for these aspect ratios modifications brought by the first 3D stationary unstable modes are not important enough to alter the transition scenario, observed in $2 \mathrm{D}$ cases, that vertical boundary layers become unstable to traveling waves to give birth to time-dependent flows. Although the unstable mode, traveling waves, should cope with the fact that the base solution is not $2 \mathrm{D}$ but $3 \mathrm{D}$, they display mainly $2 \mathrm{D}$ features.

Finally, note that the 3D numerical simulations are very time consuming. One 
period at $A_{x}=1$ needs 17960 time steps and for $A_{x}=3$ it needs 12135 time steps. Even for larger aspect ratios, computation at one Rayleigh number may also need thousands of dimensionless time units. This fact prevents from searching systematically for multiple time-dependent flows. Several examples of time evolutions are given in Figure 12.

\section{Summary and concluding remarks}

In this study was first investigated linear stability of 2D steady natural convection flows in air-filled differentially heated cavities of aspect ratios from 1 to 7 with adiabatic horizontal walls, with respect to $3 \mathrm{D}$ periodic perturbations. On the one hand, 3D periodic unstable modes are found to be stationary and lead to pitchfork bifurcations and on the other hand they are anti-centro-symmetric and break the centro-symmetry of the 2D base solution. The 3D unstable modes are concentrated in the cavity top and bottom parts and are related to the curving structures of the main flow. In curving flow structures, centrifugal force plays an important role and can result in secondary flows. It is believed that the instability mechanism is centrifugal-flow instability. In comparison with previous 2D works, only for aspect ratios 7 and 8, 2D perturbations are more dangerous than the 3D periodic ones. For other aspect ratios, 3D periodic stationary perturbations are more dangerous. This suggests that the onset of $2 \mathrm{D}$ time-dependent flows is not meaningful for most of the aspect ratios considered if there is enough space in the periodic $y$ direction and that 2D assumption should be used with caution.

3D nonlinear simulations have been performed at slightly supercritical Rayleigh numbers and indicate that the pitchfork bifurcations are surpercritical. Furthermore steady-state solutions after bifurcations are weak in $y$ direction: $v$ velocity is one order of magnitude smaller than $u$ and $w$ velocities.

Further numerical simulations have been performed at higher $R a$ in order to understand if these weak flows in $y$ direction affect the onset of time-dependent flows. For $A_{x} \leq 3$ it is these weak 3D flows, in form of internal hydraulic jump, that become unstable to perturbations of very low frequency: wave period, in the order of 100 time units, is much longer than those of the $2 \mathrm{D}$ unstable modes. The onset of time-dependent flows is due to Hopf bifurcations for $A_{x}=1$ and 3 and due to a supercritical drift pitchfork bifurcation for $A_{x}=2$. For larger $A_{x}$ (from 4 to 7 ), it is the vertical boundary layers that become unstable to Tollmien-Schlichting waves traveling along the cavity walls. Both the critical Rayleigh numbers and the structures of the unstable modes are similar to what have been observed in $2 \mathrm{D}$ cases. But the unstable modes should cope with the fact that the base solution is no more 2D. For these aspect ratios the weak $3 \mathrm{D}$ flow persists and it is not important enough to change the transition scenario that traveling waves in the vertical boundary layers are responsible for the onset of time-dependent flows.

Let's finally stress that, in order to perform this kind of studies, a number of numerical tools are necessary: a nonlinear 2D time-stepping code (the 2D unsteady incompressible Navier-Stokes equations), a 2D steady-state solving code combined with 
quadratic extrapolation, a linearized 2D/3D time-stepping code associated with Arnold's method, a steady-state solving code working on the eigensystem and using continuation method, and a 3D periodic time-stepping code (the 3D periodic unsteady Navier-Stokes equations). The basic tool is the 2D time-stepping code on which the $2 \mathrm{D}$ steady-state solving code using Newton's iteration is based. The interesting idea is to use Arnoldi's method to estimate the leading eigenpairs, determine them accurately by the steadystate code solving the eigensystem and using continuation method, and calculate the critical point using a secant method. The combination of these tools is shown to be efficient in studying stability of $2 \mathrm{D}$ natural convection flows with respect to both $2 \mathrm{D}$ and 3D perturbations. This methodology can also be applied to other flow types.

\section{Acknowledgments}

Computations have been performed at Institut du Développement des Ressources Informatique et Scientifique (IDRIS) under research projects 10326 and 11474.

\section{References}

Albensoeder S, Kuhlmann H \& Rath H 2001 Phys. Fluids 13, 121.

Bernardi C \& Maday Y 1992 in 'Collection Mathématiques \& Applications' Springer Verlag Berlin. Boyd J 2000 Chebyshev and Fourier Spectral Methods Dover publication New York.

Canuto C, Hussaini M, Quarteroni A \& Zang T 1988 Spectral methods in fluid dynamics Springer Verlag New York.

de Gassowski G, Xin S \& Daube O 2003 C. R. Acad. Sci., Ser. II b 331, 705-711.

Guermond J L \& Quartapelle L 1998 Numer. Math. 80(5), 207-238.

Haidvogel D \& Zang T 1979 J. Comp. Phys. 30, 167-180.

Keller H B 1977 in P. H Rabinowitz, ed., 'Applications of Bifurcation Theory' Academic Press New York pp. 359-384.

Labrosse G, Tric E, Khallouf H \& Betrouni M 1997 Num. Heat Trans. B 31, 261-276.

Le Quéré P 1987 Etude de la transition à l'instationnarité des écoulements de convection naturelle en cavité verticale différentiellement chauffée par méthodes spectrales Chebyshev Thèse d'Etat Univ. de Poitiers, France.

Le Quéré P \& Behnia M 1998 J. Fluid Mech. 359, 81-107.

Mamun C \& Tuckerman L 1995 Phys. Fluids 7(1), 80-91.

Mercader I, Batiste O, Ramirez-Piscin L, Ruiz X, Rudiger \& Casademunt J 2005 Phys. Fluids 17(10), 104108-.

Paolucci S \& Chenoweth D 1989 J. Fluid Mech. 201, 379-410.

Peyret R 2000 in 'Applied Mathematical Sciences' Springer Verlag New York.

Ramanan N \& Homsy G 1994 Phys. Fluids 6(8), 2690-2701.

Tuckerman L \& Barkley D 2000 in 'Numerical Methods for Bifurcation Problems and Large-Scale Dynamical Systems' Vol. 119 Springer Bologna pp. 453-466.

Winters K 1987 J. Heat Trans. 109, 894-.

Xin S \& Le Quéré P 1995 J. Fluid Mech. 304, 87-118.

Xin S \& Le Quéré P 2001 Phys. Fluids 13(9), 2529-2542.

Xin S \& Le Quéré P 2002 Int. J. Num. Meth. in Fluids 40(8), 981-998.

Xin S \& Le Quéré P 2006 Num. Heat Trans. A 50(5), 437-466.

Xin S, Le Quéré P \& Daube O 1997 Phys. Fluids 9(4), 1014-1033. 